\title{
Radial Fracture in a Three-Phase Composite: Application to Wellbore Cement Liners at Early Ages
}

\author{
Thomas Petersen, Franz-Josef Ulm* \\ Massachusetts Institute of Technology, 77 Massachusetts Avenue, Cambridge, MA 02139
}

\begin{abstract}
Little understanding exists between the early-age stress developments in a wellbore cement sheath and its risk of impairment. During hydration, the cement morphology and pore-pressure changes induce eigenstresses in the solid and pore volumes. Utilizing these stresses as the driving mechanism of fracture, this paper formalizes the inspection of a radial crack in an elastic cement sheath constrained by an inner steel casing and an outer rock formation. The solution is constructed in the framework of analytic function theory and seeks the Green's function for an edge dislocation in the intermediate cement phase. A dislocation pile-up along the line of fracture constructs a singular integral equation for the crack opening displacement derivative, from which the energy release rate is readily deduced.

Under the uniform development of eigenstresses, the stiffness ratios of steelto-cement and rock-to-cement generally predict the crack to initiate along the steel-cement interface. Here, the impacts of (i) a rigid bond and (ii) a sliding interface with no shear are assessed. This leads to the primary result of the paper: the potential for radial fracture is substantially mitigated by ensuring the shear connection between the steel casing and the cement sheath.

Keywords: Wellbore cement sheath, Radial crack, Complex variable method, Chemo-poro mechanics
\end{abstract}

\footnotetext{
${ }^{*}$ Corresponding author, Tel.: +1 6172533544

Email addresses: tapeter@mit.edu (Thomas Petersen), ulm@mit.edu (Franz-Josef Ulm)
}

Preprint submitted to Engineering Fracture Mechanics

October 21, 2015 


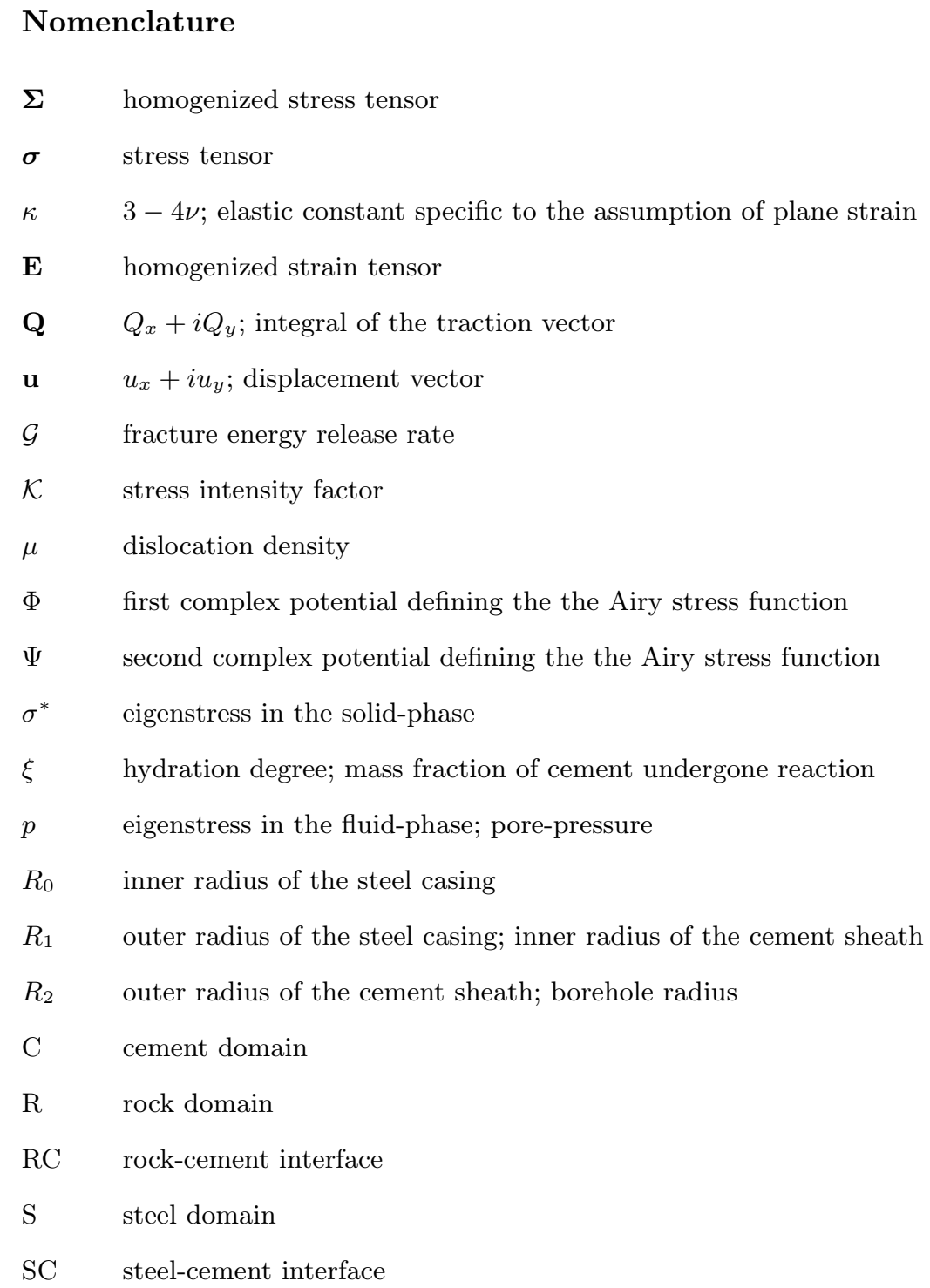




\section{Problem Statement}

In the construction of an oil or natural gas well, the cement sheath is placed between the geologic formation and the steel casing to seal reservoir fluids from overlying and underlying strata. Loss of the sealing function carries harmful

5 environmental consequences, creates potential hazards in rig and oil well operations, and assumes loss of revenue due to decreased production capacity and expensive repair operations $[1,2]$. Nonetheless, recent evidence suggests that current design practices are insufficient in safeguarding against interzonal flow. For instance, studies have indicated that leakages of methane into groundwater aquifers during unconventional gas production (i.e., gas production by hydraulic fracturing) are the result of impaired cement casings $[3,4,5]$. Though the debonding of the interfaces and the cracking of the sheath provide the most ostensible pathways for fluid migration, failure criteria have yet to be defined in the context of fracture criteria. Moreover, it is known that early-age shrinkage phenomena and pore-pressure developments are primary contributors to sheath failure [6], yet contemporary modeling efforts rarely and inadequately incorporate their physics. In this work, a framework is developed to connect the chemo-poro-elastic nature of cement to the risk of radial fracture in a wellbore cement sheath.

Cement shrinkage causes tensile hoop stresses that concentrate along the steel-cement boundary (SC) [7]. As formation fluid accumulates in open fractures, it acts to propagate cracks upward, and by extension, radially outward [8]. In our analysis, we calculate the energy release rate for a unit depth of the sheath due to a single crack emanating along SC. Several other studies have solved similar problems. Bowie and Freese provided the stress intensity factor for an edge crack in a circular ring with a uniform tension applied along the external boundary [9]. Delale and Erdogan produced the stress intensity factor for a radial crack in a hollow cylinder [10]. The solutions closest to the problem at hand were given by Luo and Chen [11] and Ardakani and Ulm [12], who solved for the energy release rate of a crack in the intermediate matrix of a 
three-phase composite cylinder. The analysis outlined in the following synthesizes and amends elements of the aforementioned works, integrates the sheath as a chemo-poro-mechanics material, and expands upon the boundary conditions posed along the cement interfaces. The result is a low-input model that respects

35 the constitutive behavior of cement and draws upon analytic function theory to assess the risk of radial fracture.

\section{Fracture Mechanics in Chemo-Porous Media}

It is now generally agreed that the mechanical behavior of cement is well described by elastic poromechanics models [13, 14, 15]. More recent models

40 capture the couplings between the eigenstress in the calcium-silicate-hydrate gel (the primary constituent of the solid cement phase) $\sigma^{*}(\xi)$, the pore-pressure development $p(\xi)$, and the deformation of the porous skeleton [16]. At early ages, the cement has a solid-to-pore volume fraction that varies in function of the hydration degree, $\xi$. This means that the growth of the solid matter requires

45 the loading of the system to be assessed incrementally and at constant hydration degree. That is, the incremental loading due to the eigenstresses, $\delta \sigma^{*}(\xi)$ and $\delta p(\xi)$, and at an instant $\xi$ in the reaction process is applied to an instantaneous system volume $V(\xi)$. A subsequent increment in loading $\left(\delta \sigma^{*}(\xi+\delta \xi) ; \delta p(\xi+\right.$ $\delta \xi))$ must be framed as a new boundary value problem whose solid volume has increased to $V(\xi+\delta \xi)$. As such, the risk of fracture is specific to the hydration degree and eigenstresses prevailing at the time of evaluation.

The energy release rate,

$$
\left.\mathcal{G}(\Gamma)\right|_{\xi, \sigma^{*}, p}=\left.\frac{\partial \mathcal{W}}{\partial \Gamma}\right|_{\xi, \sigma^{*}, p}=\frac{1}{2} \int_{\Gamma}[(\boldsymbol{\sigma}+p \mathbf{I}) \cdot \mathbf{n}] \cdot \frac{\partial \llbracket \mathbf{u} \rrbracket}{\partial \Gamma} \mathrm{d} \Gamma,
$$

is inspected by holding $\xi, \sigma^{*}$, and $p$ constant and measures the work $\partial \mathcal{W}$ required to create an additional unit of crack surface area $\partial \Gamma$. A drained fracture process will reduce the traction vector normal the surface of the crack from its initial value $\boldsymbol{\sigma} \cdot \mathbf{n}$ to its progressed value $-p \mathbf{I} \cdot \mathbf{n}$, causing a jump in displacement $\llbracket \mathbf{u} \rrbracket$. Comparing $\mathcal{G}$ to the evolution of the fracture toughness $\mathcal{G}_{\mathrm{cr}}$, which can be 


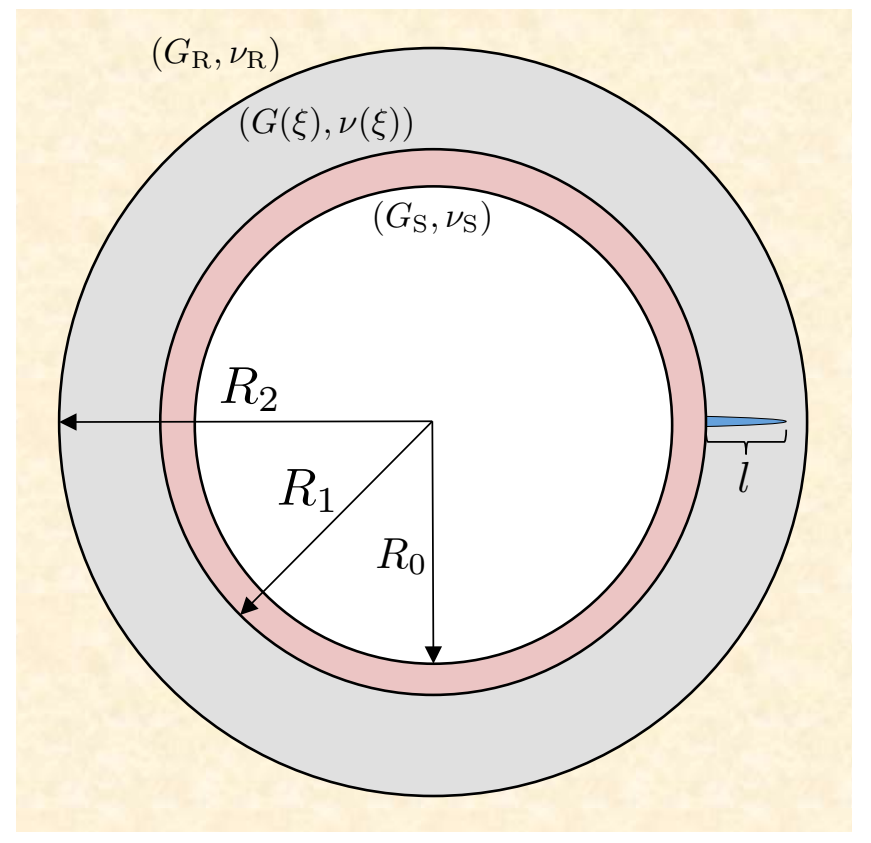

Figure 1: Diagram of a wellbore cement system with a radial crack emanating from SC

measured experimentally (See, e.g. Ref. [14]), allows the margin of safety from radial fracture to be evaluated in progression of the early-age loading, where an

60 equality of the relation

$$
\left.\mathcal{G}\right|_{\xi, \sigma^{*}, p} \leq\left.\mathcal{G}_{\mathrm{cr}}\right|_{\xi}
$$

substantiates the risk of an advance of the crack.

\section{Definition of the Boundary Value Problem}

The geometry of the wellbore system is depicted in Fig.1 and is modeled as three isotropic material domains bounded by concentric circular contours in the complex plane, $z=r e^{i \theta}$. The steel casing is placed with its center at the origin and has an inner radius of $R_{0}$ and an outer radius of $R_{1}$. Surrounding the steel casing, the cement sheath is bounded at its inner radius by $|z|=R_{1}$ and at its outer radius by $|z|=R_{2}$. Finally, the rock formation borders the exterior of the cement at $|z|=R_{2}$ and extends indefinitely to $z \rightarrow \infty$. We denote the material 
The incremental constitutive relations of the three materials is written as,

$$
\begin{aligned}
& \delta \boldsymbol{\Sigma}(z, \xi)=\mathbb{C}_{\mathrm{S}}: \delta \mathbf{E}(z, \xi) \quad z \in \mathrm{S} \\
& \delta \boldsymbol{\Sigma}(z, \xi)=\mathbb{C}_{\mathrm{C}}(\xi): \delta \mathbf{E}(z, \xi)+\delta \boldsymbol{\Sigma}^{*}(\xi) \quad z \in \mathrm{C} \\
& \delta \boldsymbol{\Sigma}(z, \xi)=\mathbb{C}_{\mathrm{R}}: \delta \mathbf{E}(z, \xi)+\delta p(z, \xi) \mathbf{I} \quad z \in \mathrm{R}
\end{aligned}
$$

where $\mathbb{C}_{S}, \mathbb{C}_{C}$, and $\mathbb{C}_{R}$ are the stiffness tensors of the steel, cement, and rock, respectively. Assuming isotropic, homogenizable materials, the stiffness tensors can be described by the shear moduli and Poisson ratios, such that $\mathbb{C}_{S}=f\left(G_{\mathrm{S}}, \nu_{\mathrm{S}}\right), \mathbb{C}_{C}=f(G(\xi), \nu(\xi))$, and $\mathbb{C}_{R}=f\left(G_{\mathrm{R}}, \nu_{\mathrm{R}}\right)$. Because the cement a poro-elastic solid, its bulk parameters $G(\xi)$ and $\nu(\xi)$ depend on $\xi$. Fig. 2 plots the evolution of the poro-elastic parameters for a typical Class $\mathrm{G}$ oil well cement.

Next, $\delta \mathbf{E}$ denotes the elastic strain due to the boundary restraints, and $\delta \boldsymbol{\Sigma}^{*}(\xi)=f\left(\delta \sigma^{*}(\xi), \delta p(\xi)\right)$ is the effective eigenstress acting in the bulk cement specimen. In our analysis, $\delta \boldsymbol{\Sigma}^{*}$ is assumed to develop uniformly in the cement. It should be noted that the porous nature of the adjacent rock prompts an equal change in pressure $\delta p(z)$ along the cement-rock boundary, diminishing as $z \rightarrow \infty$.

The incremental development of the effective eigenstress in the cement requires the stress state to be evaluated under consideration of the elastic responses of the steel and the rock. Hence, the plane elastic problem may be solved by imposing continuity conditions of traction and displacement along the 
interfaces, and a pressure boundary condition along the crack surface:

$$
\begin{aligned}
\delta \boldsymbol{\Sigma}\left(R_{0}, \theta\right) \cdot \mathbf{n} & =\mathbf{0} & & \theta \in[0,2 \pi] \\
\llbracket \delta \boldsymbol{\Sigma}\left(R_{1}, \theta\right) \cdot \mathbf{n} \rrbracket & =\mathbf{0} & & \theta \in[0,2 \pi] \\
\llbracket \delta \mathbf{u}\left(R_{1}, \theta\right) \rrbracket & =\mathbf{0} & & \theta \in[0,2 \pi] \\
\llbracket \delta \boldsymbol{\Sigma}\left(R_{2}, \theta\right) \cdot \mathbf{n} \rrbracket & =\mathbf{0} & & \theta \in[0,2 \pi] \\
\llbracket \delta \mathbf{u}\left(R_{2}, \theta\right) \rrbracket & =\mathbf{0} & & \theta \in[0,2 \pi] \\
\delta \boldsymbol{\Sigma}(r \rightarrow \infty) & =\underline{\underline{\mathbf{0}}} & & \theta \in[0,2 \pi] \\
\delta \Sigma_{\theta \theta} & =\delta p & & r \in\left[p_{1}, p_{2}\right] ; \theta=0
\end{aligned}
$$

Above, $\mathbf{n}$ is the unit normal and $\llbracket \cdot \rrbracket$ denotes the jump in the vector quantity across the interface. The crack length $l$ extends along the real axis from $p_{1}=R_{1}$ to $p_{2}=R_{1}+l$, where $l \leq R_{2}-R_{1}$. As the radial fracture is predicted to originate along the steel-cement interface (SC), the condition of tangential displacement continuity, Eq.(4c), creates a shear stress singularity at $z=p_{1}$, maintaining the closed shape of the crack. Because the stress concentration near the proximal crack tip will cause damage to the steel-cement bond in a physical system, we investigate the radial fracture under a second set of boundary conditions where Eq.(4b) and Eq.(4c) are replaced by,

$$
\begin{array}{rrr}
\llbracket \delta \boldsymbol{\Sigma}\left(R_{1}, \theta\right) \cdot \mathbf{n} \rrbracket \cdot \mathbf{e}_{r}=0 & \theta \in[0,2 \pi] \\
\left(\delta \boldsymbol{\Sigma}^{+}\left(R_{1}, \theta\right) \cdot \mathbf{n}\right) \cdot \mathbf{e}_{\theta} & \\
=\left(\delta \boldsymbol{\Sigma}^{-}\left(R_{1}, \theta\right) \cdot \mathbf{n}\right) \cdot \mathbf{e}_{\theta}=0 & \theta \in[0,2 \pi] \\
\llbracket \delta \mathbf{u}\left(R_{1}, \theta\right) \rrbracket \cdot \mathbf{e}_{r}=\mathbf{0} . & \theta \in[0,2 \pi]
\end{array}
$$

${ }_{85}$ Herein, ${ }^{+}$and ${ }^{-}$indicate values upon approaching the boundary from the left and right sides, respectively ${ }^{1}$. These modifications allow the steel and cement to slip along one another without shear, while maintaining radial contact.

\footnotetext{
${ }^{1} \mathrm{As}$ is conventional, the left and right sides are defined for a counterclockwise traversal of the contour.
} 


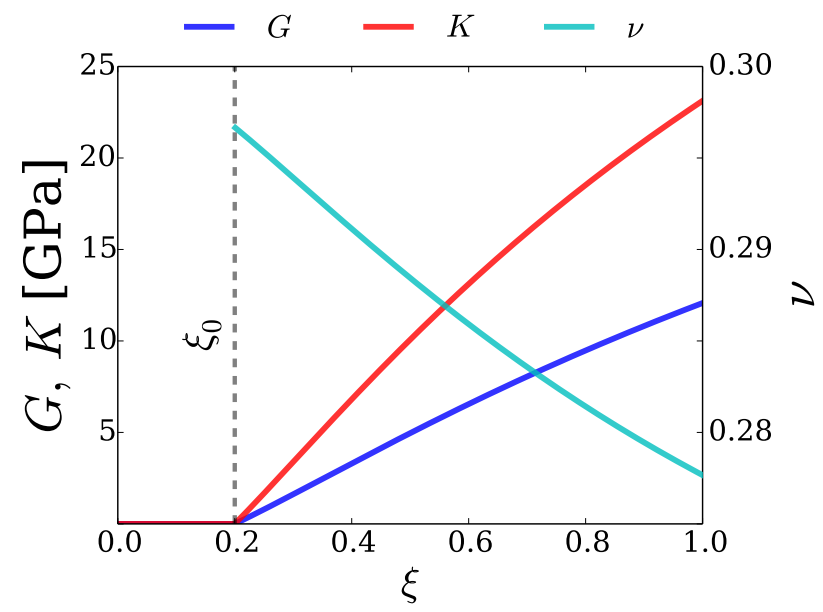

Figure 2: Simulated evolution of the shear modulus $G$, bulk modulus $K$, and Poisson's ratio $\nu$ of hydrating wellbore cement

The general solution to the elastic stress state of the cement sheath is separated into two sub-problems:

90

(i) A continuous cement annulus, absent of defects, with tangential stress $\sigma_{\theta \theta}$ evolving due to the uniform development of eigenstresses and pressure changes, and

(ii) a sectionally holomorphic annulus with a crack of length $l$, where an effective stress $\Sigma_{\theta \theta}=\sigma_{\theta \theta}+p$ acts on the crack surfaces. The effective stress must be opposite in sign and equal in magnitude (less the added pressure term) to the stress evaluated in the defect-free system. The superposition establishes a hydrostatic pressure along the crack lips, consistent with a drained fracture process.

Hence, the elastic states of (ii) before and after the advancement of the crack can be compared to determine the energy release rate in Eq.(1). 


\section{Stress State in a Continuous Cement Sheath}

The elastic stress in the continuous sheath in (i) is readily deduced from the well known solution for a tube with elastic boundary responses (see, e.g. Ref. [17]). Due to the porous nature of the adjacent rock and the non-porous boundary of the steel, the loading originating in the solid and pore spaces of the cement affect the structural response along these two interfaces differently. A uniform development of eigenstresses develops an effective tangential stress of the form,

$$
\begin{aligned}
& \delta \Sigma_{\theta \theta}\left(\xi_{i}\right)=\delta \sigma_{\theta \theta}\left(\xi_{i}\right)+\delta p\left(\xi_{i}\right)=D_{1}\left(\xi_{i}\right)+\frac{D_{2}\left(\xi_{i}\right)}{r^{2}} \\
& \Sigma_{\theta \theta}\left(\xi_{t}\right) \approx I_{1}\left(\xi_{t}\right)+\frac{I_{2}\left(\xi_{t}\right)}{r^{2}}=\sum_{i \leq t}\left[D_{1}\left(\xi_{i}\right)+\frac{D_{2}\left(\xi_{i}\right)}{r^{2}}\right]
\end{aligned}
$$

where the parameters must account for $\sigma^{*}$ and $p$ separately,

$$
D_{k}\left(\xi_{i}\right)=g_{k}\left(\xi_{i}\right) \delta \sigma_{i}^{*}+h_{k}\left(\xi_{i}\right) \delta p_{i} . \quad k=1,2
$$

The presence of a single radial crack along the thickness of the sheath entails the loss of radial symmetry of the problem, requiring the stress state of (ii) to be solved as a function of both $r$ and $\theta$. Employing the theory of complex variables, we proceed by calculating the Green's function for an edge dislocation in the cement domain. The stress state is then evaluated by integrating the Green's function along the line of the fracture, respecting the pressure boundary condition along the crack surface. 


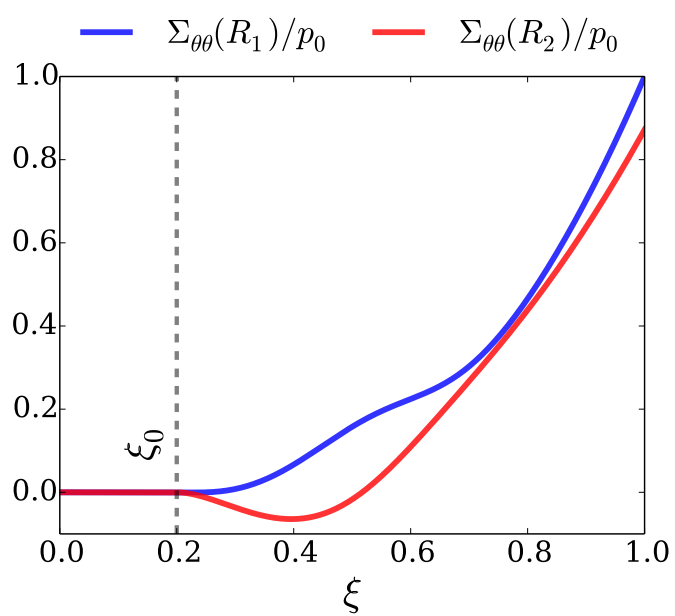

Figure 3: Simulated evolution of the effective hoop stress $\Sigma_{\theta \theta}=\sigma_{\theta \theta}+p$ along SC (blue) and $\mathrm{RC}$ (red), normalized by a reference stress $p_{0}=9.07 \mathrm{MPa}$

\subsection{Analytic Representation of Stress and Displacement States}

The Muskhelishvili representation of the in-plane displacement vector $\mathbf{u}$ and stress tensor $\boldsymbol{\Sigma}$ for an isotropic material under plane strain $(\kappa=3-4 \nu)$ are given by[18],

$$
\begin{aligned}
2 G\left(u_{r}+i u_{\theta}\right) & =e^{-i \theta}\left(\kappa \Phi(z)-z \overline{\Phi^{\prime}(z)}-\overline{\Psi(z)}\right) \\
i \int \boldsymbol{\Sigma} \cdot \mathbf{n} \mathrm{d} z & =\Phi(z)+z \overline{\Phi^{\prime}(z)}+\overline{\Psi(z)}+C \\
\Sigma_{r r}+i \Sigma_{r \theta} & =\overline{\Phi^{\prime}(z)}+\Phi^{\prime}(z)-e^{2 i \theta}\left(\bar{z} \Phi^{\prime \prime}(z)+\Psi^{\prime}(z)\right) \\
\Sigma_{\theta \theta}+i \Sigma_{r \theta} & =\overline{\Phi^{\prime}(z)}+\Phi^{\prime}(z)+e^{2 i \theta}\left(\bar{z} \Phi^{\prime \prime}(z)+\Psi^{\prime}(z)\right)
\end{aligned}
$$

where $z=x+i y, \Phi(z)$ and $\Psi(z)$ are analytic, $\Phi^{\prime}(z)=\mathrm{d} \Phi / \mathrm{d} z, C$ is an arbitrary constant, and the overbar denotes complex conjugation. For an annular region, $\Phi(z)$ can be continued across the inner and outer boundaries $\left(|z|=r_{1}\right.$ and $\left.|z|=r_{2}\right)$ to extend its analytic domain[19]:

$$
\Phi(z)= \begin{cases}-z \overline{\Phi^{\prime}}\left(r_{1}^{2} / z\right)-\bar{\Psi}\left(r_{1}^{2} / z\right) & \text { if } r_{1}^{2} / r_{2} \leq|z|<r_{1} \\ \Phi(z) & \text { if } r_{1} \leq|z| \leq r_{2} \\ -z \overline{\Phi^{\prime}}\left(r_{2}^{2} / z\right)-\bar{\Psi}\left(r_{2}^{2} / z\right) & \text { if } r_{2}<|z| \leq r_{2}^{2} / r_{1}\end{cases}
$$




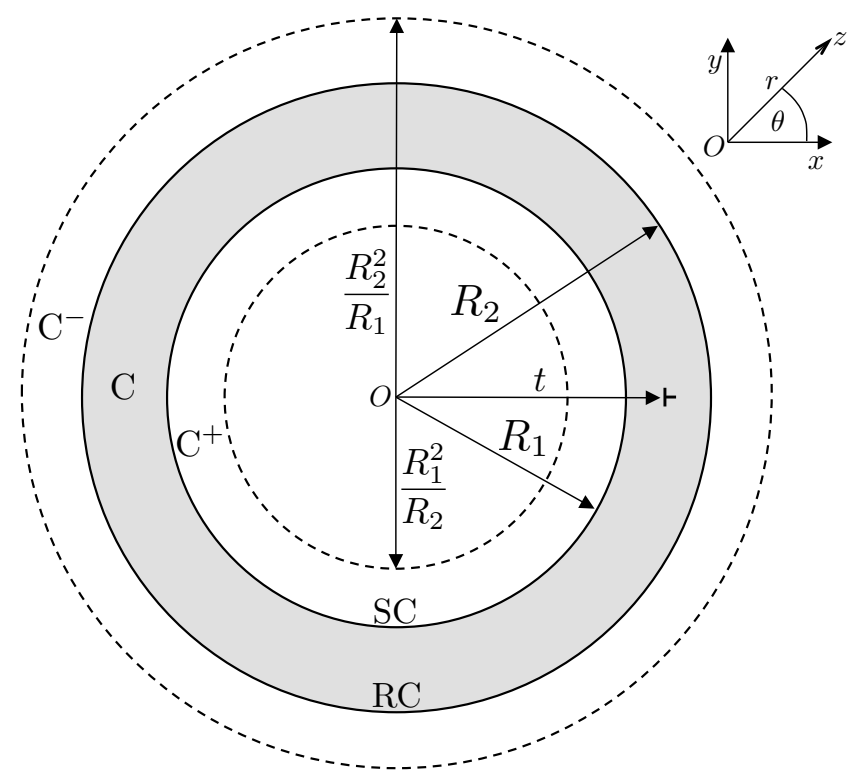

Figure 4: Continuation of the annular region of the cement domain across $\mathrm{SC}$ and $\mathrm{RC}$

The continuation of the cement domain is visualized in Fig.4, where $\mathrm{C}^{+}$and $\mathrm{C}^{-}$ indicate the interior and exterior extensions, respectively. Similar continuations are applied to $\mathrm{S}$ and $\mathrm{R}$. The stress and displacement equations are therefore given in a simplified form:

$$
\begin{aligned}
& 2 G\left(u_{r}+i u_{\theta}\right)=e^{-i \theta}\left(\kappa \Phi(z)+\Phi\left(r_{d}^{2} / z\right)\right) \\
& Q_{x}+i Q_{y}=\int(\boldsymbol{\Sigma} \cdot \mathbf{n}) \mathrm{d} z=-i\left[\Phi(z)-\Phi\left(r_{d}^{2} / z\right)+C\right]
\end{aligned}
$$

Eqn.(9), require a compatibility condition for $\Psi(z)$,

$$
\bar{\Phi}\left(r_{2}^{2} / z\right)-\bar{\Phi}\left(r_{1}^{2} / z\right)+\frac{r_{2}^{2}-r_{1}^{2}}{z} \Phi^{\prime}(z)=0
$$

to fully define the cement mechanical state.

\subsection{Green's Function for an Edge Dislocation}

The Green's function, used to model a radial crack in the cement sheath, 135 solves for a divergence free stress state with a delta inhomogeneity positioned 
at $z=t$, where $z \in C$. Here, the delta inhomogeneity describes the effects of an edge dislocation. Mathematically, this means that the traversal around the inhomogeneity in $\mathrm{C}$ produces an in-plane jump in displacement that is proportional to the strength of the dislocation. For the application of determining the fracture energy release rate, a continuous arrangement of discontinuities models the crack opening displacement- sometimes referred to as dislocation pile-up. Hence, a gradient of dislocation strengths is positioned in the sheath to recreate the shape of the crack. The great utility of the Green's function is that, once at hand, it can be used to construct any arbitrarily shaped crack in the region $V=\mathrm{C}$

A solution to the Green's function will be sought in two steps by adopting an approach akin to the procedure used in Eshelby's inclusion problem[20]:

(1) In the first step, the stress state due to an edge dislocation embedded in an unconstrained cement medium will be denoted by $\boldsymbol{\Sigma}^{(1)}=\boldsymbol{\Sigma}^{\inf }+\boldsymbol{\Sigma}^{*}$. By calculating $Q_{x}^{\mathrm{inf}}+i Q_{y}^{\mathrm{inf}}$ along $\mathrm{SC}$ and $\mathrm{RC}$ due to an edge dislocation in an infinite, homogeneous medium, the deformation of an unconstrained cement annulus is determined by applying an equal and opposite loading along the sheath boundaries and measuring the holomorphic response $\Sigma^{*}$.

(2) Next, the surface traction, defined by $\tilde{Q}_{x}+i \tilde{Q}_{y}$, is chosen to restore the boundaries into their original position. Here, a variation in the tangential displacement $u_{\theta}$ along SC is admitted in the case of a slip boundary. By placing the cement sheath into the cavity between steel and rock, and allowing the system to relax, the interfacial response of the steel and the rock, $\hat{\Sigma}$, is calculated. The combined result of the restoration and relaxation of the boundaries is recorded as $\Sigma^{(2)}=\tilde{\Sigma}+\hat{\Sigma}$.

The superposed result of (1) an edge dislocation in an unconstrained sheath, and (2) the placement of the sheath into the cavity between steel and rock produce the stress field due to an edge dislocation in a constrained specimen, in the form:

$$
\boldsymbol{\Sigma}^{\mathrm{dis}}=\boldsymbol{\Sigma}^{(1)}+\boldsymbol{\Sigma}^{(2)}
$$


165 A solution to $\Sigma^{(1)}$ and $\Sigma^{(2)}$ is sought by developing $\Phi_{d}(z)$ into Laurent series for the three material regions. Consequently, a total of 8 series are required to define the mechanical state of the cemented oil well system ${ }^{2}$. In general, these can be written as

$$
\Phi_{d}(z)= \begin{cases}\sum_{n=\infty}^{\infty} a_{n}^{+} z^{n} & \text { if } z \in V^{+} \\ \sum_{n=\infty}^{\infty} a_{n} z^{n} & \text { if } z \in V \\ \sum_{n=\infty}^{\infty} a_{n}^{-} z^{n} & \text { if } z \in V^{-}\end{cases}
$$

and we define the coefficients for the three regions $d=\{\mathrm{S}, \mathrm{C}, \mathrm{R}\}$ as

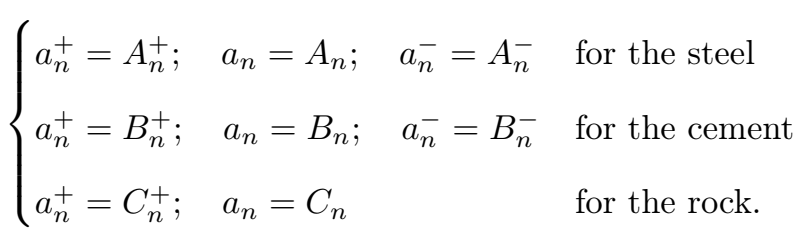

The dash across the summation symbols in Eq.(13) indicates that the zerothorder terms in the series, which describe rigid body displacements, are omitted (i.e., $a_{0}^{+}=a_{0}=a_{0}^{-}=0$ ). Furthermore, the coefficients of the cement sheath can be decomposed into three contributions:

$$
B_{n}=\underbrace{B_{n}^{*}}_{\text {remove traction }}+\underbrace{\tilde{B}_{n}}_{\text {restore boundary position }}+\underbrace{\hat{B}_{n}}_{\text {relax against S and } \mathrm{R}}
$$

\subsubsection{First Step: Unconstrained Sheath with an Edge Dislocation}

The Green's function solves the mechanical state of the cement sheath (i.e., produce an expression for $\Phi(z))$ for an edge dislocation with a Burgers vector of unit value $\left|b_{x}+i b_{y}\right|=1$ placed at $z=t$. Because the desired result is a radial crack along a single ray (where $\theta$ is constant) and the displacement jump shall occur perpendicular to the crack, the result is simplified by assuming the position of the dislocation along the real axis $(\Im(t)=0)$ with a Burgers vector pointing in the $\mathrm{y}$-direction $\left(b_{x}=0\right)$. Under these simplifications, the potentials

\footnotetext{
${ }^{2} 3$ series to represent $\Phi(z)$ in $\mathrm{C}^{+}, \mathrm{C}$, and $\mathrm{C}^{-}, 3$ series to represent $\Phi_{\mathrm{S}}(z)$ in $\mathrm{S}^{+}, \mathrm{S}$, and $\mathrm{S}$, and 2 series to represent $\Phi_{\mathrm{R}}(z)$ in $\mathrm{R}^{+}$, and $\mathrm{R}$.
} 
for a dislocation in an infinite cement medium are well known and given as [21],

$$
\begin{array}{ll}
\Phi^{\inf }(z)=\frac{G}{\pi(\kappa+1)} \log (z-t) & z \in \mathrm{C} \\
\Psi^{\inf }(z)=\frac{G}{\pi(\kappa+1)}\left(\log (z-t)-\frac{z}{z-t}\right) & z \in \mathrm{C}
\end{array}
$$

175 It should be noted that $\Phi^{\inf }(z)$ and $\Psi^{\inf }(z)$ are singular at $z=t$, and that additional singularities exist in the image regions at $z=R_{1}^{2} / t$ where $z \in \mathrm{C}^{+}$ and $z=R_{2}^{2} / t$ where $z \in C^{-}$.

It follows that the stress resultant of an arc along the boundaries $r=|z|=$ $\left\{R_{1}, R_{2}\right\}$ is calculated by Eq.(10b) as:

$$
\begin{aligned}
& i \mathbf{Q}^{\mathrm{inf}}=i\left[Q_{x}^{\mathrm{inf}}+i Q_{y}^{\mathrm{inf}}\right] \\
= & \begin{cases}\Omega\left(\log (z-t)+\log \left(\frac{z-R_{1}^{2} / t}{z}\right)+\left(\frac{R_{1}^{2}-z^{2}}{t}\right) \frac{1}{z-R_{1}^{2} / t}\right) & z \in \mathrm{SC} \\
\Omega\left(\log (z-t)+\log \left(\frac{z-R_{2}^{2} / t}{z}\right)+\left(\frac{R_{2}^{2}-z^{2}}{t}\right) \frac{1}{z-R_{2}^{2} / t}\right) & z \in \mathrm{RC}\end{cases}
\end{aligned}
$$

where $\Omega=\frac{G}{\pi(\kappa+1)}$.

Because the mechanical state of the wellbore system will be resolved in terms of Laurent series, the effect of the singularities in Eq.(17) must similarly be decomposed into powers of $z^{n}$. The following important power series expansions are utilized:

$$
\begin{array}{ll}
\log (1-z)=\sum_{n=1}^{\infty}-\frac{z^{n}}{n} & \text { for }|z|<1 \\
\frac{1}{1-z}=\sum_{n=0}^{\infty} z^{n} & \text { for }|z|<1
\end{array}
$$

Recognizing that $|z|<t$ and $|z|>R_{1}^{2} / t$ along SC and $|z|>t$ and $|z|<R_{2}^{2} / t$ along RC, the expressions in Eq.(17) are recast as,

$$
\begin{aligned}
i \mathbf{Q}^{\inf }(z) & =\sum_{n=1}^{\infty} \Omega\left(\frac{-1}{n t^{n}} z^{n}+\left(\frac{n-1}{n}\right)\left(\frac{R_{1}^{2}}{t}\right)^{n} z^{-n}\right) \\
& -\sum_{n=-1}^{\infty} \Omega\left(\frac{R_{1}^{(2 n+2)}}{t^{(n+2)}} \frac{1}{z^{n}}\right)=\sum_{-\infty}^{\infty} \alpha_{n}^{*} z^{n} \quad z \in \mathrm{SC}
\end{aligned}
$$




$$
\begin{aligned}
i \mathbf{Q}^{\inf }(z) & =\sum_{n=1}^{\infty} \Omega\left(\frac{-t^{n}}{n} z^{-n}-\left(\frac{n+1}{n}\right)\left(\frac{t}{R_{2}^{2}}\right)^{n} z^{n}\right) \\
& +\sum_{n=2}^{\infty} \Omega\left(\frac{t^{(n-2)}}{R_{2}^{(2 n-2)}} z^{n}\right)=\sum_{-\infty}^{\infty} \beta_{n}^{*} z^{n} \quad z \in \mathrm{RC}
\end{aligned}
$$

The boundary value problem of a cement annulus free from its steel and

185 the Supplemental Information. Here, the coefficients $B_{n}^{*+}, B_{n}^{*}$, and $B_{n}^{*-}$ resolve the stress state in the cement region and its continuations. Hence, the potentials defining the stress in the sheath with an edge dislocation and traction-free surfaces are written as:

$$
\Phi^{(1)}(z)= \begin{cases}-z \overline{\Phi^{\inf }}\left(R_{1}^{2} / z\right)-\overline{\Psi^{\inf }}\left(R_{1}^{2} / z\right)+\Sigma_{-\infty}^{\infty} B_{n}^{*+} z^{n} & z \in \mathrm{C}^{+} \\ \Phi^{\inf }(z)+\Sigma_{-\infty}^{\infty} B_{n}^{*} z^{n} & z \in \mathrm{C} \\ -z \overline{\Phi^{\inf }}\left(R_{2}^{2} / z\right)-\overline{\Psi^{\inf }}\left(R_{2}^{2} / z\right)+\Sigma_{-\infty}^{\infty} B_{n}^{*-} z^{n} & z \in \mathrm{C}^{-}\end{cases}
$$

\subsubsection{Second Step: Placement into Cavity Between Steel and Rock}

Next, we calculate the boundary responses of the steel casing and the rock formation by placing the deformed cement sheath into the undeformed cavity between the steel and rock. Starting with the mechanical state of the sheath at the end of the first step, the displacements along the boundaries that must be restored are given by:

$$
\begin{aligned}
& u_{r}^{(1)}+i \chi u_{\theta}^{(1)} \\
= & \frac{(1+\chi) e^{-i \theta}}{4 G}\left[\kappa \Phi^{(1)-}(z)+\Phi^{(1)+}(z)\right] \\
+ & \frac{(1-\chi) e^{i \theta}}{4 G}\left[\kappa \overline{\Phi^{(1)-}(z)}+\overline{\Phi^{(1)+}(z)}\right] \quad z \in \mathrm{SC} \\
& u_{r}^{(1)}+i u_{\theta}^{(1)} \\
= & \frac{e^{-i \theta}}{2 G}\left(\kappa \Phi^{(1)+}(z)+\Phi^{(1)-}(z)\right) \quad z \in \mathrm{RC}
\end{aligned}
$$

where the tangential displacement along SC is included (excluded) by setting $\chi=1(\chi=0)$, depending on the slip condition. 
By prescribing equal and opposite displacements unto the sheath, the relevant traction resultant is determined by,

$$
i \tilde{\mathbf{Q}}(z)= \begin{cases}\sum_{-\infty}^{\infty} \tilde{B}_{n} z^{n}-\sum_{-\infty}^{\infty} \tilde{B}_{n}^{+} z^{n} & z \in \mathrm{SC} \\ \sum_{-\infty}^{\infty} \tilde{B}_{n} z^{n}-\sum_{-\infty}^{\infty} \tilde{B}_{n}^{-} z^{n} & z \in \mathrm{RC}\end{cases}
$$

where $\tilde{B}_{n}, \tilde{B}_{n}^{+}$, and $\tilde{B}_{n}^{-}$are given in the Supplemental Information. In the case of a sliding inclusion, the coefficients were chosen to produce zero tangential stresses along SC.

Finally, the steel and rock material boundaries are added, the cement sheath is inserted into the cavity, and the interfacial response is calculated due to the tractions along SC and RC defined by $\tilde{\mathbf{Q}}$. We may ensure satisfaction of the boundary conditions (Eqn.(4a-f), resp. Eqn.(4a,d-f) and Eqn.(5a-c)) using the following relations (all quantities refer to the cement sheath unless $\mathrm{S}_{\mathrm{S}}$ or $\mathrm{R}$ indicate reference to the steel or rock.):

(1) Traction-free conditions along the inner surface of the steel casing $\left(z_{0}=\right.$ $\left.{ }_{205} R_{0} e^{i \theta} ; 0<\theta<2 \pi\right)$ :

$$
\hat{\Phi}_{\mathrm{S}}^{+}\left(z_{0}\right)-\hat{\Phi}_{\mathrm{S}}^{-}\left(z_{0}\right)=0
$$

(2) Traction continuity along SC $\left(z_{1}=R_{1} e^{i \theta} ; 0<\theta<2 \pi\right)$ :

$$
\hat{\Phi}_{\mathrm{S}}^{+}\left(z_{1}\right)-\hat{\Phi}_{\mathrm{S}}^{-}\left(z_{1}\right)=\hat{\Phi}^{-}\left(z_{1}\right)-\hat{\Phi}^{+}\left(z_{1}\right)+i \tilde{\mathbf{Q}}\left(z_{1}\right)
$$

(3) Displacement continuity along $\mathrm{SC}\left(z_{1}=R_{1} e^{i \theta} ; 0<\theta<2 \pi\right)$ :

$$
\left[\kappa_{\mathrm{S}} \hat{\Phi}_{\mathrm{S}}^{+}\left(z_{1}\right)+\hat{\Phi}_{\mathrm{S}}^{-}\left(z_{1}\right)\right]=\frac{G_{\mathrm{S}}}{G}\left[\kappa \hat{\Phi}^{-}\left(z_{1}\right)+\hat{\Phi}^{+}\left(z_{1}\right)\right]
$$

or

Radial displacement continuity along $\mathrm{SC}\left(z_{1}=R_{1} e^{i \theta} ; 0<\theta<2 \pi\right)$ :

$$
\begin{aligned}
& {\left[\kappa_{\mathrm{S}} \hat{\Phi}_{\mathrm{S}}^{+}\left(z_{1}\right)+\hat{\Phi}_{\mathrm{S}}^{-}\left(z_{1}\right)\right]+\frac{z_{1}^{2}}{R_{1}^{2}}\left[\kappa_{\mathrm{S}} \overline{\hat{\Phi}_{\mathrm{S}}^{+}\left(z_{1}\right)}+\overline{\hat{\Phi}_{\mathrm{S}}^{-}\left(z_{1}\right)}\right] } \\
= & \frac{G_{\mathrm{S}}}{G}\left(\left[\kappa \hat{\Phi}^{-}\left(z_{1}\right)+\hat{\Phi}^{+}\left(z_{1}\right)\right]+\frac{z_{1}^{2}}{R_{1}^{2}}\left[\kappa \overline{\hat{\Phi}^{-}\left(z_{1}\right)}+\overline{\hat{\Phi}^{+}\left(z_{1}\right)}\right]\right),
\end{aligned}
$$


and loss of shear traction along $\mathrm{SC}\left(z_{1}=R_{1} e^{i \theta} ; 0<\theta<2 \pi\right)$ :

$$
\begin{aligned}
& {\left[\hat{\Phi}_{\mathrm{S}}^{\prime}{ }^{+}\left(z_{1}\right)+\overline{\hat{\Phi}_{\mathrm{S}}^{\prime}-\left(z_{1}\right)}\right]-\frac{z_{1}^{4}}{R_{1}^{4}}\left[\overline{\hat{\Phi}_{\mathrm{S}}^{\prime}\left(z_{1}\right)}+\hat{\Phi}_{\mathrm{S}}^{\prime}-\left(z_{1}\right)\right] } \\
= & {\left[\hat{\Phi}^{\prime}+\left(z_{1}\right)+\overline{\hat{\Phi}^{\prime}-\left(z_{1}\right)}\right]-\frac{z_{1}^{4}}{R_{1}^{4}}\left[\overline{\hat{\Phi}^{\prime}+\left(z_{1}\right)}+\hat{\Phi}^{\prime}-\left(z_{1}\right)\right] }
\end{aligned}
$$

(4) Traction continuity along $\mathrm{RC}\left(z_{2}=R_{2} e^{i \theta} ; 0<\theta<2 \pi\right)$ :

$$
\hat{\Phi}^{+}\left(z_{2}\right)-\hat{\Phi}^{-}\left(z_{2}\right)+f^{(1)}=\hat{\Phi}_{\mathrm{R}}^{-}\left(z_{2}\right)-\hat{\Phi}_{\mathrm{R}}^{+}\left(z_{2}\right)+i \tilde{\mathbf{Q}}\left(z_{2}\right)
$$

(5) Displacement continuity along $\mathrm{RC}\left(z_{2}=R_{2} e^{i \theta} ; 0<\theta<2 \pi\right)$ :

$$
\left[\kappa \hat{\Phi}^{+}\left(z_{2}\right)+\hat{\Phi}^{-}\left(z_{2}\right)\right]=\frac{G}{G_{\mathrm{R}}}\left[\kappa_{\mathrm{R}} \hat{\Phi}_{\mathrm{R}}^{-}\left(z_{2}\right)+\hat{\Phi}_{\mathrm{R}}^{+}\left(z_{2}\right)\right]
$$

(6) Zero far-field effective stress condition in the formation. This boundary condition truncates the positive powers of the Laurent series for $\Phi_{\mathrm{R}}(z)$ in $z \in \mathrm{R}$ and the negative powers in $z \in \mathrm{R}^{+}$to ensure a finite value at $z=\infty$ and $z=0$, respectively.

The task that remains is the assembly of a system of equations that solves for the Laurent series coefficients in $\hat{\Phi}_{d} ; d=\{\mathrm{S}, \mathrm{C}, \mathrm{R}\}$, where $\hat{B}_{n}^{+}, \hat{B}_{n}$, and $\hat{B}_{n}^{-}$ are the final coefficients needed to calculate the Green's function in Eq.(12). Again, the Supplemental Information provides details on this procedure.

\section{Singular Integral Equation for the Dislocation Density}

The Green's function derived in the previous section solves the stress state in the cement sheath for a single edge dislocation with a unit Burgers vector pointing in the $y$-direction. Due to the linearity of the response, the influence of a dislocation of variable strength $\mu$ - commonly termed the dislocation density is measured by $\mu \boldsymbol{\Sigma}^{\text {dis }}$. The versatility of the function is immediately recognized: By relating the dislocation density to the crack opening displacement, a radial crack of variable penetration and origin can be constructed.

While devising the circumstances of a radial crack for a known distribution of the dislocation density $\mu(t)$ is trivial, the risk of radial fracture for the 
hydrating cement sheath must be assessed with respect to the poromechanical properties and the eigenstress generation. Consequently, we are tasked with finding the distribution that relates the tangential stress generation in a holomorphic specimen to the crack surface traction in the impaired specimen (i.e., Eq.(6)). From the above, the hoop stress generated at $x=r$ by a dislocation at $x=t$ is resolved as

$$
\begin{aligned}
& \mu(t) \Sigma_{\theta \theta}^{\operatorname{dis}}(r, t)=\mu(t)\left(\Sigma_{\theta \theta}^{(1)}(r, t)+\Sigma_{\theta \theta}^{(2)}(r, t)\right) \\
= & \mu(t)\left(\Sigma_{\theta \theta}^{\inf }(r, t)+\Sigma_{\theta \theta}^{*}(r, t)+\tilde{\Sigma}_{\theta \theta}(r, t)+\hat{\Sigma}_{\theta \theta}^{*}(r, t)\right)
\end{aligned}
$$

where the tangential stress is calculated by Eq.(10d):

$$
\Sigma_{\theta \theta}(r, t)=\Re\left\{2 \Phi^{\prime}(r, t)+r \Phi^{\prime \prime}(r, t)+\Psi^{\prime}(r, t)\right\} .
$$

Therefore,

$$
\mu(t) \Sigma_{\theta \theta}^{\inf }(r, t)=\frac{2 G}{\pi(\kappa+1)} \frac{\mu(t)}{r-t}
$$

and the holomorphic part can be represented as,

$$
\mu(t)\left(\Sigma_{\theta \theta}^{*}(r, t)+\tilde{\Sigma}_{\theta \theta}(r, t)+\hat{\Sigma}_{\theta \theta}^{*}(r, t)\right)=\mu(t) H(r, t) .
$$

Remembering that $\Psi(z, t)$ is calculated by either of the cases in Eq.(9), we choose

$$
\begin{aligned}
\Psi(r, t) & =-\Phi\left(R_{1}^{2} / r, t\right)-\frac{R_{1}^{2}}{r} \Phi^{\prime}(r, t) \\
& =-\sum_{n=-\infty}^{\infty} B_{n}^{+}(t) R_{1}^{2} r^{-n}-\sum_{n=-\infty}^{\infty} n B_{n}(t) R_{1}^{2} r^{n-1} .
\end{aligned}
$$

Whence, it follows from Eq.(32) and Eq.(15):

$$
\begin{aligned}
H(r, t) & =\sum_{n=-\infty}^{\infty} B_{n}(t)\left[\left(n^{2}+n\right) r^{n-1}-\left(n^{2}-2 n\right) R_{1}^{2} r^{n-3}\right] \\
& +\sum_{n=-\infty}^{\infty} B_{n}^{+}(t)\left[n R_{1}^{2 n} r^{-n-1}\right] .
\end{aligned}
$$

The above kernel $H(r, t)$ measures the tangential stress at location $r$ due to the boundary response of an edge dislocation of unit strength at location $t$, where 
$r \in\left[R_{1}, R_{2}\right]$ and $t \in\left(R_{1}, R_{2}\right)$. With the relevant expressions at hand, the surface traction along the crack in Eq.(6) is guaranteed by setting

$$
\underbrace{\frac{2 G}{\pi(\kappa+1)} \int_{p_{1}}^{p_{2}} \frac{\mu(t)}{t-r} d t}_{\text {dislocations in } \infty \text { medium }}+\underbrace{\int_{p_{1}}^{p_{2}} H(r, t) \mu(t) d t}_{\mathrm{S} \text { and } \mathrm{R} \text { boundaries }}=-\left(I_{1}+\frac{I_{2}}{r^{2}}\right) .
$$

We notice that $\mu(t)$ can be solved as a singular integral equation of the first kind, where the integrals must be taken in the Cauchy Principal Value sense ${ }^{3}$.

\subsection{Numerical Solution to the Integral Equation}

Much work has been devoted to the analysis of singular integral equations [18], and noteworthy contributions by Erdogan et al. [22] give numerical approaches to the solution of crack-type problems. In particular, they have adopted Gaussian quadrature formulas that utilize the orthogonality of the Jacobi polynomials to provide high-accuracy solutions with a small number of quadrature points. Here, $\mu(t)$ is sought as

$$
\mu(t)=w(t) g(t)=\left(t-p_{1}\right)^{\lambda_{1}}\left(p_{2}-t\right)^{\lambda_{2}} g(t)
$$

and $w(t)$ is the pre-determined weight function. It depends on two parameters, $\lambda_{1}$ and $\lambda_{2}$, that describe the nature of the crack tip singularities. Because a Burgers vector is defined as the integral of $\partial u_{i} / \partial s$ taken counter-clockwise around the dislocation, it is readily understood that

$$
\mu(r)=\frac{\partial \llbracket u_{y} \rrbracket}{\partial r}
$$

defines the derivative of the crack opening displacement. If a closed crack is embedded in a homogeneous medium, $\mu(t)$ will be singular at the points $t=p_{1}$ and $t=p_{2}$. For this scenario, the symmetry of the top and bottom crack surfaces

\footnotetext{
${ }^{3}$ E.g., the Cauchy principal value of an integral $\int_{a}^{b} f(x) d x$ - also termed the finite part of an integral - with a singularity at the end point $x=b$ may be calculated as

$$
\text { P.V. } \int_{a}^{b} f(x) d x=\lim _{\epsilon \rightarrow 0^{+}} \int_{a}^{b-\epsilon} f(x) d x .
$$


and the elliptical shape requires the opening displacement to terminate at an

recast as

$$
\begin{aligned}
& \frac{1}{\pi} \int_{-1}^{1} \frac{\hat{\mu}(\tau)}{\tau-\rho} d \tau+\frac{1}{2} \int_{-1}^{1} \hat{H}(\rho, \tau) \hat{\mu}(\tau) d \tau \\
= & -\left(\hat{I}_{1}+\frac{\hat{I}_{2}}{r(\rho)^{2}}\right)
\end{aligned}
$$

where

$$
\begin{aligned}
& \hat{H}(\rho, \tau)=\frac{(\kappa+1)\left(p_{2}-p_{1}\right)}{2 G} H(r(\rho), t(\tau)) \\
& \hat{I}_{1}=\frac{(\kappa+1)}{2 G} I_{1} ; \quad \hat{I}_{2}=\frac{(\kappa+1)}{2 G} I_{2} \\
& \hat{\mu}(\tau)=\hat{w}(\tau) \hat{g}(\tau)=(\tau-1)^{\lambda_{1}}(1-\tau)^{\lambda_{2}} \hat{g}(\tau)
\end{aligned}
$$

and the coordinates $r$ and $t$ are parameterized using the following relations:

$$
\begin{gathered}
r(\rho)=\frac{p_{2}-p_{1}}{2} \rho+\frac{p_{2}+p_{1}}{2} \\
t(\tau)=\frac{p_{2}-p_{1}}{2} \tau+\frac{p_{2}+p_{1}}{2} .
\end{gathered}
$$

\subsubsection{Closed Crack Geometry: Rigid Shear Connection Along SC}

The first case we consider is that of a rigid connection between steel and cement, for which an elliptical crack shape enables tangential displacement continuity across SC. While a crack tip embedded in a homogeneous medium has a square-root singularity in $w(t)$, the weight function takes a different form in the case that either of the crack tips terminates at a bi-material interface. Here, a shear stress singularity along $\mathrm{SC}$ and/or RC maintains the closure of the crack. As a result, the Fredholm kernel $\hat{H}(\rho, \tau)$ in Eq.(41) is singular for $\rho=\tau= \pm 1$, whence $\lambda_{1} \neq-1 / 2$ and $/$ or $\lambda_{2} \neq-1 / 2$. 
It was shown in Ref.[23] that the degree of the singularity $\lambda$ in the weight function for a crack originating in an elastic medium $a$ and terminating perpendicular to the interface of a second medium $b$ can be calculated from

$$
\cos (\lambda \pi)=\frac{2\left(\Delta_{2}-\Delta_{1}\right)}{1+\Delta_{2}}(1+\lambda)^{2}+\frac{\Delta_{1}+\Delta_{2}^{2}}{1-\Delta_{2}^{2}} \quad \lambda<1
$$

where

$$
\begin{aligned}
& \Delta_{1}=\frac{G_{a}\left(1-\nu_{b}\right)-G_{b}\left(1-\nu_{a}\right)}{G_{a}\left(1-\nu_{b}\right)+G_{b}\left(1-\nu_{a}\right)} \\
& \Delta_{2}=\frac{G_{a}\left(1-2 \nu_{b}\right)-G_{b}\left(1-2 \nu_{a}\right)}{G_{a}\left(1-\nu_{b}\right)+G_{b}\left(1-\nu_{a}\right)}
\end{aligned}
$$

are the Dunders' parameters $[24]^{4}$.

By substituting the expression for $\hat{\mu}(\tau)$ in Eq.(42c) into Eq.(41) and discretizing $\hat{g}(\mu)$, a system of linear algebraic equations is developed using a Gauss-Jacobi quadrature scheme. Polyanin and Manzhirov give a numerical method that is readily adopted to estimate the equation at hand([26]; Chapter 15):

$$
\begin{aligned}
\sum_{i=1}^{N} w_{i} \hat{g}\left(\tau_{i}\right)\left[\frac{1}{\tau_{i}-\rho_{k}}+\frac{\pi}{2} \hat{H}\left(\rho_{k}, \tau_{i}\right)\right] & =-\left(\hat{I}_{1}+\frac{\hat{I}_{2}}{r\left(\rho_{k}\right)^{2}}\right) \\
(k & =1,2,3, \ldots, N-1) .
\end{aligned}
$$

Herein, the abscissas and weights are approximated to high accuracy by,

$$
\begin{array}{rlrl}
\tau_{i} & \approx \cos \left(\theta_{i}\right) ; & \theta_{i} & =\frac{\pi}{2}\left(\frac{2 \lambda_{1}-1+4 i}{2 N+\lambda_{1}+\lambda_{2}+1}\right) \\
\rho_{k} & \approx \cos \left(\vartheta_{k}\right) ; \quad \vartheta_{k}=\frac{\pi}{2}\left(\frac{2 \lambda_{1}+1+4 k}{2 N+\lambda_{1}+\lambda_{2}+1}\right) \\
w_{i} & \approx \frac{2}{2 N+\lambda_{1}+\lambda_{2}+1} \sqrt{1-\tau_{i}^{2}}\left(1-\tau_{i}\right)^{\lambda_{1}}\left(1+\tau_{i}\right)^{\lambda_{2}}
\end{array}
$$

if $N \gg 1$. If both ends of the crack have integrable singularities, $\lambda_{1}$ and $\lambda_{2}$ are in the range $(-1,0]$ and $\cos \left(\vartheta_{k}\right)$ provides only $N-1$ abscissas in the interval $[1,-1]$. The final equation is obtained from the desired result that the opening

\footnotetext{
${ }^{4}$ While the result was initially deduced for a crack terminating perpendicular to a flat interface, it was later confirmed to extend to cracks terminating at a circular bi-material interface (e.g., see Ref.[25])
} 

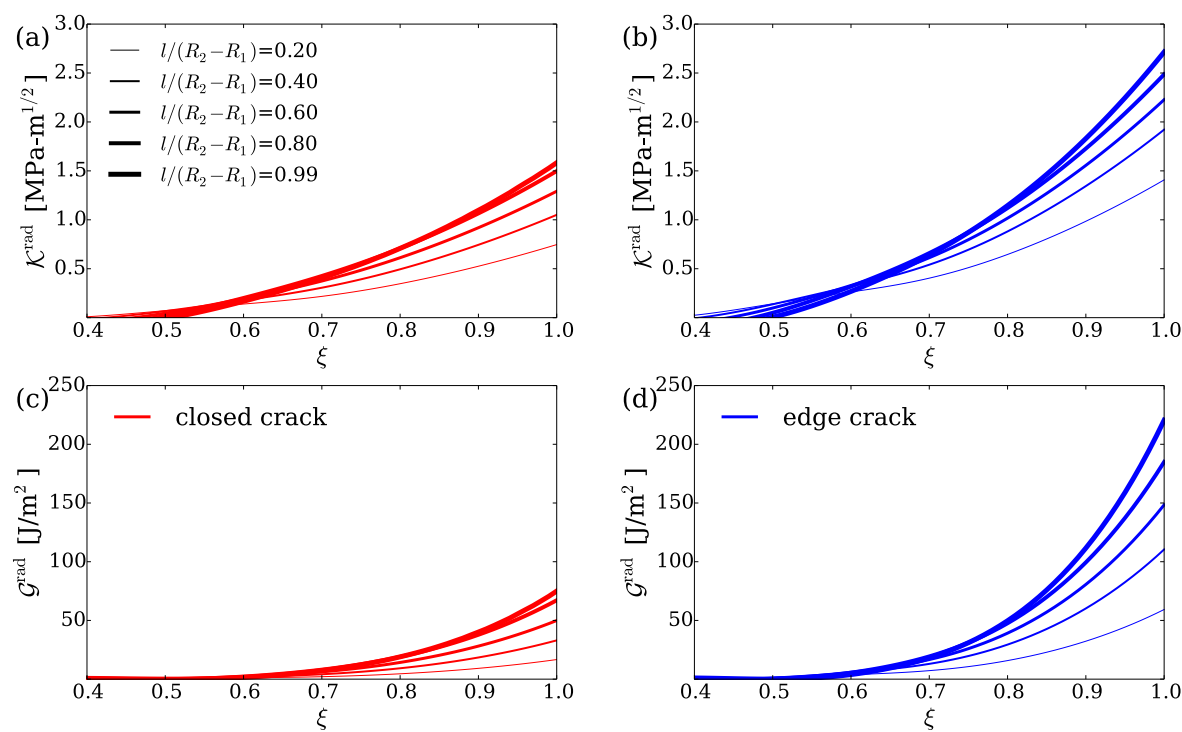

Figure 5: The evolution of (a)(b) the stress intensity factor and (c)(d) the energy release rate during the hydration reaction for (a)(c) the open crack geometry, and (b)(d) the closed crack geometry. The weight of the lines indicates the extension of the radial crack through the sheath thickness.

displacement is zero at both ends of the crack:

$$
\int_{-1}^{1} \hat{\mu}(\tau) \mathrm{d} \tau \approx \sum_{i=1}^{N} w_{i} \hat{g}\left(\tau_{i}\right)=0
$$

\subsubsection{Open Crack Geometry: Complete Loss of Shear Along SC}

In the event that the shear stress between steel and cement is finite, the radial crack will assume a greater opening displacement and, consequently, also a greater stress intensity factor. Where the proximal end of the crack is otherwise restrained from opening, the loss of the interfacial bond allows the crack to open unimpeded. More precisely, the displacement jump across SC is measured as the aggregated contribution of the dislocation pile-up: $\int_{p_{1}}^{p_{2}} \mu(t) d t$. The curves

in Fig.6 depict the resulting surface displacements for an open crack.

Gupta and Erdogan [27] proved analytically that the weight function of an 
Table 1: Model verification of the normalized stress intensity factor for an edge crack (columns 1 - 3) and an embedded crack (columns $4-6$ ) in a hollow cylinder (i.e., $G_{\mathrm{S}}=G_{\mathrm{R}} \rightarrow 0$ ) with a uniform crack surface pressure of $P$ and $R_{1}=R_{2} / 2$. The embedded crack has a constant length of $l=0.5\left(R_{2}-R_{1}\right)$ and $\mathcal{K}$ is calculated for the distal tip. The reference values have been adopted from Ref. [10]; $N=50$ and positive indices of the Laurent series are truncated at the values provided in the parentheses.

\begin{tabular}{ccc|ccc}
$\frac{\left(p_{2}-R_{1}\right)}{\left(R_{2}-R_{1}\right)}$ & $\frac{\mathcal{K}_{\text {edge }}^{\mathrm{Ref}}}{P \sqrt{l}}$ & $\frac{\mathcal{K}_{\text {edge }}}{P \sqrt{l}}$ & $\frac{\left(p_{1}-R_{1}\right)}{\left(R_{2}-R_{1}\right)}$ & $\frac{\mathcal{K}_{\text {embed }}^{\text {Ref }}}{P \sqrt{l / 2}}$ & $\frac{\mathcal{K}_{\text {embed }}}{P \sqrt{l / 2}}$ \\
\hline$\rightarrow 0$ & 1.122 & $1.115(600)$ & 0.05 & n.a. & $1.256(300)$ \\
0.1 & 1.157 & $1.154(400)$ & 0.15 & 1.176 & $1.175(200)$ \\
0.2 & 1.247 & $1.247(400)$ & 0.20 & 1.178 & $1.177(200)$ \\
0.3 & 1.366 & $1.364(400)$ & 0.25 & 1.198 & $1.197(200)$ \\
0.4 & 1.503 & $1.503(200)$ & 0.30 & 1.239 & $1.238(200)$ \\
0.5 & 1.658 & $1.657(200)$ & 0.35 & 1.312 & $1.311(200)$ \\
0.6 & 1.830 & $1.831(100)$ & 0.40 & n.a. & $1.446(200)$ \\
0.7 & 2.030 & $2.031(100)$ & 0.45 & n.a. & $1.767(200)$
\end{tabular}

edge crack takes the form,

$$
\hat{w}(\tau)=\frac{1}{\sqrt{1-\tau}}
$$

The equation above ensures the finite slope of the surface displacement upon nearing the steel interface. The square root singularity signifies that the crack tip is embedded in a homogeneous medium. Now, it is desirable to extend the definition of $\hat{\mu}(\tau)$ beyond the physical extent of the domain in order to make use of the Gaussian integration formula with the orthogonal Chebyshev polynomials. As was done in Ref. [27], we proceed by defining the following normalization of the radial coordinate,

$$
\tau(t)=\frac{t-p_{1}}{p_{2}-p_{1}} \quad \rho(r)=\frac{r-p_{1}}{p_{2}-p_{1}}
$$

such that a continuation across $\tau=0$ can be made,

$$
\hat{\mu}(\tau)=\frac{\hat{g}(\tau)}{\sqrt{(1-\tau)}} ; \quad \hat{g}(\tau)=\hat{g}(-\tau) \quad(-1<\tau<1) .
$$



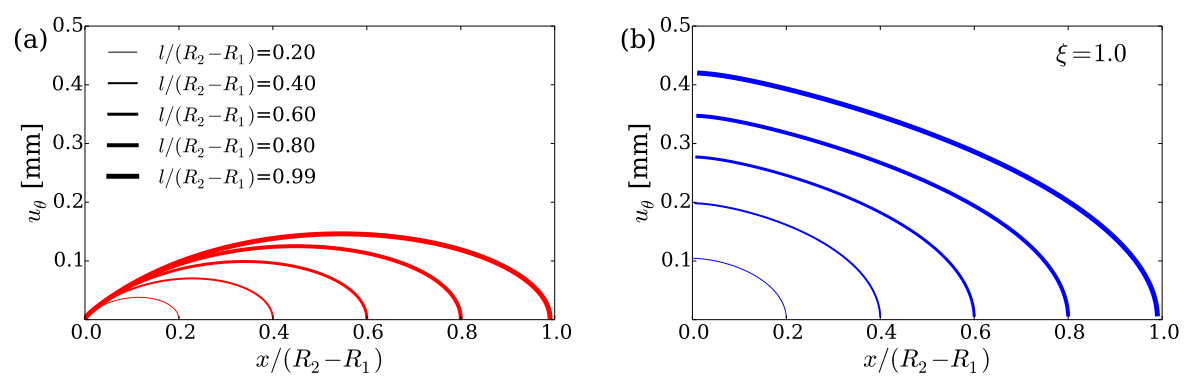

Figure 6: Plots of the surface displacement of the radial crack, $\frac{1}{2} \llbracket u_{\theta} \rrbracket$, for the assumptions of (a) shear continuity and (b) complete loss of shear along SC for varying degree of crack extension and at complete hydration.

and the integral equation in Eq.(41) is recast as

$$
\begin{aligned}
& \frac{1}{\pi} \int_{0}^{1} \frac{\hat{g}(\tau)}{\sqrt{1-\tau}}\left[\frac{1}{\tau-\rho}+\hat{H}(\rho, \tau)\right] d \tau \\
& \frac{1}{2 \pi} \int_{-1}^{1} \frac{\hat{g}(\tau)}{\sqrt{1-\tau^{2}}}\left[\frac{1}{|\tau|-\rho}+\hat{H}(\rho,|\tau|)\right] d \tau \\
= & -\left(\hat{I}_{1}+\frac{\hat{I}_{2}}{r(\rho)^{2}}\right)
\end{aligned}
$$

The procedure for solving the integral equation with $\hat{w}(\tau)=1 / \sqrt{1-\tau^{2}}$ is readily adopted from Ref.[22]:

$$
\begin{aligned}
& \frac{1}{2} \sum_{i=1}^{2 N+1} \frac{1}{2 N+1} \hat{g}\left(\tau_{i}\right)\left[\frac{1}{\left|\tau_{i}\right|-\rho_{k}}+\pi \hat{H}\left(\rho_{k},\left|\tau_{i}\right|\right)\right] \\
= & \sum_{i=1}^{N} \frac{1}{2 N+1} \hat{g}\left(\tau_{i}\right)\left[\frac{1}{\tau_{i}-\rho_{k}}+\pi \hat{H}\left(\rho_{k}, \tau_{i}\right)\right] \\
= & -\left(\hat{I}_{1}+\frac{\hat{I}_{2}}{r\left(\rho_{k}\right)^{2}}\right)
\end{aligned}
$$

where the abscissas are calculated from,

$$
\begin{array}{cc}
\tau_{i}=\cos \left(\pi \frac{2 i-1}{4 N+2}\right) & (i=1,2,3, \ldots N) \\
\rho_{k}=\cos \left(\frac{\pi k}{2 N+1}\right), & (k=1,2,3, \ldots N)
\end{array}
$$

and it is recognized that the summation at $\tau_{m}=0$ vanishes due to symmetry. 
The formal definition of the mode I stress intensity factor for a crack whose tip is embedded in a homogeneous medium is given by

$$
\mathcal{K}=\lim _{r \rightarrow p_{2}} \sqrt{2 \pi\left(p_{2}-r\right)} \Sigma_{\theta \theta}(r, \theta=0),
$$

where $\Sigma_{\theta \theta}(r, \theta=0)$ is the effective stress acting ahead of the crack. It is well established that the singular stress behavior in proximity of the crack tip may be expressed in terms of the cleavage stress $\varsigma_{\theta \theta}$, which takes the form [22],

$$
\begin{aligned}
\left.\varsigma_{\theta \theta}(r)\right|_{r>p_{2}} & =\frac{2 G}{1+\kappa}\left|\frac{\partial u_{\theta}(r)}{\partial r}\right|_{r<p_{2}}+O\left[\left(r-p_{2}\right)^{\eta}\right] \\
& \approx \frac{2 G}{1+\kappa}|\mu(r)|_{r<p_{2}}, \quad(\eta>0)
\end{aligned}
$$

Thus, the stress intensity factor can be rewritten as

$$
\begin{aligned}
\mathcal{K} & =\lim _{r \rightarrow p_{2}} \sqrt{2 \pi\left(p_{2}-r\right)} \varsigma_{\theta \theta} \\
& =\frac{2 G}{1+\kappa} \lim _{r \rightarrow p_{2}} \sqrt{2 \pi\left(p_{2}-r\right)}|\mu(r)|
\end{aligned}
$$

and after substituting $\mu(r)=g(r)\left(r+p_{1}\right)^{\lambda_{1}} / \sqrt{p_{2}-r}$, where $g(r)=(l / 2)^{\frac{1}{2}-\lambda_{1}} \hat{g}(\tau(r))$, into the equation above we arrive at,

$$
\mathcal{K}=\frac{2 G}{1+\kappa} \sqrt{\pi l} 2^{\lambda_{1}}|\hat{g}(1)| .
$$

The value of $\lambda_{1}$ has been left ambiguous and depends on whether the proximal tip adheres to an open or closed geometry, though the final expression for $\mathcal{K}$ coincides. Stress intensity factors were calculated for embedded cracks, and edge cracks where $G_{\mathrm{S}}$ and $G_{\mathrm{R}}$ were set to zero to resemble a "hollow" ring. Their solution compared well with the results given in [10] (see Table 1).

Finally, the surface displacement of the crack is obtained by

$$
\left.u_{\theta}(r, 0)=-\frac{1}{2} \int_{x}^{p_{2}} \mu(r) d r\right\} p_{1}<r<p_{2}
$$

and is readily calculated at the collocation points for an open crack by,

$$
u_{\theta}\left(t\left(\tau_{k}\right)\right) \approx\left(\frac{l}{2}\right) \sum_{i=1}^{k}-w_{i} \hat{g}\left(\tau_{i}\right)
$$

where the appropriate weights for the chosen numerical procedure should be substituted. 

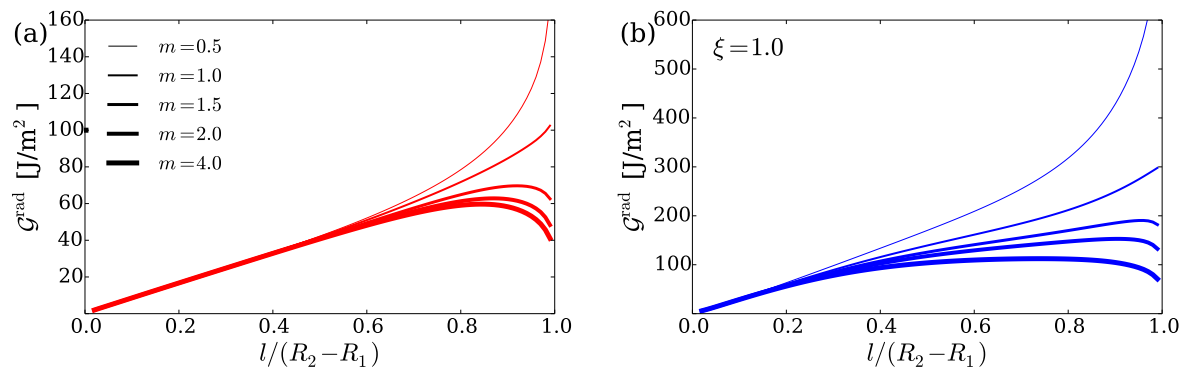

Figure 7: The energy release rate of a radial crack plotted as a function of the crack extension for the assumptions of (a) shear continuity and (b) complete loss of shear along SC. The rock shear modulus is varied between $5 \mathrm{GPa}$ and $40 \mathrm{GPa}$.

\section{Results}

The results of our radial fracture solution are assessed for typical conditions encountered during a primary cementing operation (see Table 2). Moreover, Fig. 2 provides the simulated evolution of the cement elastic properties and Fig. 3 gives the evolution of the tangential stress as calculated by Eq.(6) for a sheath in good condition. These provide the input for the results to be discussed.

The stress intensity factor for the two variants of the boundary value problem, those of a rigid connection between steel and cement and a slippery interface, are investigated in Fig. 5a and Fig. 5b. The widths of the lines indicate the depth of crack penetration and all cracks originate from SC. As expected, the loss of a tangential bond along SC increases the risk of radial cracking: the stress in the cement "flows" toward one rather than two crack tips.

To investigate the radial crack from an energy perspective, the dislocation

Table 2: Input parameters for the sample simulation.

\begin{tabular}{ccccccc}
\multicolumn{4}{c}{ Geometry $[\mathrm{cm}]$} & \multicolumn{4}{c}{ Elastic Constants $[\mathrm{GPa}] /[-]$} \\
$R_{0}$ & $R_{1}$ & $R_{2}$ & $G_{\mathrm{S}}\left(E_{\mathrm{S}}\right)$ & $\nu_{\mathrm{S}}$ & $G_{\mathrm{R}}\left(E_{\mathrm{R}}\right)$ & $\nu_{\mathrm{R}}$ \\
\hline 10 & 11 & 16 & $78.7(200)$ & 0.27 & $15.4(40)$ & 0.30
\end{tabular}




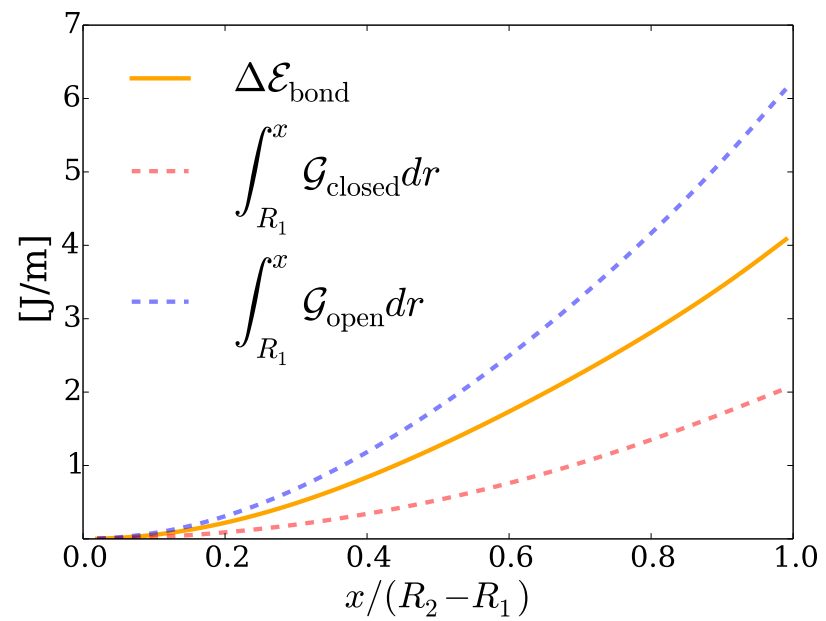

Figure 8: A comparison of the total energy released by a crack initiating from SC and propagating a length $l$ for the open (blue) and closed (red) crack geometries.

density is used in combination with Eq.(1)

$$
\mathcal{G}=\int_{p_{1}}^{p_{2}}\left(I_{1}+\frac{I_{2}}{r^{2}}\right) \mu(r) d r \leq \mathcal{G}_{\mathrm{cr}}
$$

to calculate the energy release rate per unit depth of the sheath. Thus, as displayed in Fig. 5c and Fig. 5d, the work done to infinitesimally advance the open crack at full hydration and near full penetration measures $\sim 200 \mathrm{~J} / \mathrm{m}^{2}$, compared to $\sim 75 \mathrm{~J} / \mathrm{m}^{2}$ for the closed crack. In the former, the system's compliance with respect to a stress acting along the crack surface is increased by the absence of a counteracting shear stress. Consequently, the crack opens wider, releasing more elastic potential energy $\left(\mathcal{G} \propto \int d \llbracket u_{\theta} \rrbracket\right)$. Because the energy release rate generally tends to increase monotonically as the crack advances, at least for the late hardening stage in which the whole thickness of the sheath is under tangential tension, the fracture process for the given parameters is considered unstable.

To further investigate the stability of the fracture process upon nearing $\mathrm{RC}$, Fig. 7a and Fig. 7b display $\mathcal{G}$ in function of the crack length for varying ratios of the cement-to-rock shear moduli $m=G / G_{\mathrm{R}}$. As can be expected, $m$ is a principal determinant of the crack stability, where $d \mathcal{G} / d l<0$ defines a stable 
process. If $m<1$ and the loading remains unchanged, an advancing crack may arrest before fully penetrating the sheath, while compliant rock, $m>1$, prompts further propagation. It is observed that the ratio, $m$, more noticeably impacts cracks of short length if closure is maintained along SC.

Fig. 6 displays the crack shapes of the two boundary traction conditions for increasing penetration depths. The maximum opening displacement of the open crack $(\sim 0.8 \mathrm{~mm})$ reaches around $2-3$ times that of the closed crack $(\sim$ $0.3 \mathrm{~mm}$ ). Bachu and Bennion experimented with brine and $\mathrm{CO}_{2}$ to evaluate the permeability of wellbore liners in the lab. The permeability was found to be strongly dependent on the presence microannuli and radial cracking, where apertures were recorded between $0.01 \mathrm{~mm}$ and $0.3 \mathrm{~mm}$ [28]. Though precise conditions of the mechanics driving the fracture processes in their experimental samples is unclear, it is of comfort that the results for the opening displacement of our simulation are of a similar order of magnitude.

Next, we elaborate on the implications of the shear bond condition along SC. As the open crack shape enforces a zero shear stress along steel and cement and the closed crack shape maintains a rigid connection, the potential energy released during fracture can be related to the energy stored in the shear bond. 3во In particular, the debonding energy for a radial crack that extends from $p_{1}$ to $p_{2}$ may be written as,

$$
\begin{aligned}
\Delta \mathcal{E}_{\text {bond }} & =\int_{p_{1}}^{p_{2}} \mathcal{G}_{\text {open }}-\mathcal{G}_{\text {closed }} d r \\
& =\frac{1}{2} \int_{p_{1}}^{p_{2}}\left(I_{1}+\frac{I_{2}}{r^{2}}\right)\left(\llbracket u_{\theta}^{\text {open }} \rrbracket-\llbracket u_{\theta}^{\text {closed }} \rrbracket\right) d r .
\end{aligned}
$$

Herein, we have related the energy release rate to the work done by the surface stress to open the crack by an amount $\llbracket u_{\theta} \rrbracket$. By integrating the energy release rate along the propagated line of fracture we arrive at the total energy dissipated 385 during fracture. The difference between the energy quantities for the open and closed crack geometries measures the potential energy stored in the rigid shear bond and is plotted by the orange line in Fig. 8. Remarkably, most of the energy released in the open crack geometry can be averted by improving the 
bond between steel and cement.

\section{Concluding Remark}

Though the health of wellbore cement liners is vital to the efficient extraction and ultimate recovery of fossil fuel resources, few studies have investigated the risk assessment of such liners from a physico-chemical viewpoint. In this study, poroelasticity provides a tool to understand and design cement sheaths against radial fracture in a framework that incorporates the stress developments in the cement microphases. Key to the accurate description of the crack propagation is capturing the drained nature of the fracture process. Under this condition, the saturated cement medium expels fluid into newly created fissures, which exerts additional pressure onto the crack surfaces and exacerbates the risk of failure. The ultimate intent of this work is to provide an easily accessible solution to the radial fracture that demands little user input, yet accurately models the singular behavior of the stresses near the crack tip(s); properties not guaranteed by numerical techniques. While we have restricted our investigation to cracks that initiate from SC, the derived Green's function is readily adopted for embedded cracks or cracks that initiate from RC. Additionally, it should be noted that our solution enables calculation of the energy release rate of a vertically tunneling crack by adopting the approach of Hutchinson and Suo [29], in which the energy stored in a horizontal slice far behind the crack front is compared to the energy stored in a slice far ahead of the crack front.

As a final remark, a comprehensive safety evaluation of wellbore liners needs to consider an extensive set of parameters that define geometry, elastic and fluid transport properties, and the cement mix design. This model is well adapted to proceed in a study of these parameters and has already acknowledged the toughness of the bond between material interfaces as critical to the mitigation of radial cracking. Acting to increase the compliance of the crack surfaces, the loss of shear along SC drastically increases the opening displacement and subsequent 
release of elastic potential energy upon advancing the crack tip.

\section{Acknowledgements}

This work was financially supported by the National Science Foundation Graduate Research Fellowship Program and Schlumberger. Additionally, the authors would like to thank the researchers and engineers at the SchlumbergerDoll Research Center and the Schlumberger Riboud Product Center for their thoughts shared during weekly phone conferences.

\section{References}

[1] C. E. Cooke, M. P. Kluck, R. Medrano, Field measurements of annular pressure and temperature during primary cementing, Journal of Petroleum Technology 35 (08) (1983) 1-429.

[2] K. J. Goodwin, R. J. Crook, Cement sheath stress failure, SPE Drilling Engineering 7 (4) (1992) 291-296.

[3] R. B. Jackson, A. Vengosh, T. H. Darrah, N. R. Warner, A. Down, R. J. Poreda, S. G. Osborn, K. Zhao, J. D. Karr, Increased stray gas abundance in a subset of drinking water wells near marcellus shale gas extraction, Proceedings of the National Academy of Sciences 110 (28) (2013) 1125011255.

[4] S. G. Osborn, A. Vengosh, N. R. Warner, R. B. Jackson, Methane contamination of drinking water accompanying gas-well drilling and hydraulic fracturing, Proceedings of the National Academy of Sciences 108 (20) (2011) $8172-8176$.

[5] E. Stokstad, Will fracking put too much fizz in your water?, Science 344 (6191) (2014) 1468-1471.

[6] A. Bonett, D. Pafitis, Getting to the root of gas migration, Oilfield Review 8 (1) (1996) 36-49. 
[7] T. A. Petersen, Chemo-poro-elastic fracture mechanics of wellbore cement liners: the role of eigenstress and pore pressure on the risk of fracture, Master's thesis, Massachusetts Institute of Technology (6 2015).

[8] M. Zhang, S. Bachu, Review of integrity of existing wells in relation to $\mathrm{Co}_{2}$ geological storage: What do we know?, International Journal of Greenhouse Gas Control 5 (4) (2011) 826-840.

450 [9] O. L. Bowie, C. E. Freese, Elastic analysis for a radial crack in a circular ring, Engineering Fracture Mechanics 4 (2) (1972) 315-321.

[10] F. Delale, F. Erdogan, Stress intensity factors in a hollow cylinder containing a radial crack, International Journal of Fracture 20 (4) (1982) 251-265.

[11] H. A. Luo, Y. Chen, An edge dislocation in a three-phase composite cylinder model, Journal of Applied Mechanics 58 (1) (1991) 75-86.

[12] S. M. Ardakani, F.-J. Ulm, Chemoelastic fracture mechanics model for cement sheath integrity, Journal of Engineering Mechanics 140 (4) (2013) 04013009 .

[13] F.-J. Ulm, G. Constantinides, F. H. Heukamp, Is concrete a poromechanics materials?-a multiscale investigation of poroelastic properties, Materials and structures 37 (1) (2004) 43-58.

[14] C. G. Hoover, F.-J. Ulm, Experimental chemo-mechanics of early-age fracture properties of cement paste, Cement and Concrete Research 75 (2015) $42-52$.

${ }_{465}$ [15] S. Ghabezloo, J. Sulem, S. Guédon, F. Martineau, J. Saint-Marc, Poromechanical behaviour of hardened cement paste under isotropic loading, Cement and Concrete research 38 (12) (2008) 1424-1437.

[16] F.-J. Ulm, M. Abuhaikal, T. Petersen, R. J. M. Pellenq, Poro-chemofracture-mechanics... bottom-up: Application to risk of fracture design of 
oil and gas cement sheath at early ages, Computational Modelling of Concrete Structures 1 (2014) 61.

[17] A. E. H. Love, A treatise on the mathematical theory of elasticity, Vol. 1, Cambridge University Press, 2013.

[18] N. I. Muskhelishvili, Some basic problems of the mathematical theory of elasticity.

[19] A. H. England, Complex variable methods in elasticity, Courier Corporation, 2012.

[20] J. D. Eshelby, The determination of the elastic field of an ellipsoidal inclusion, and related problems 241 (1226) (1957) 376-396.

[21] J. R. Rice, Mathematical analysis in the mechanics of fracture, Fracture: an advanced treatise 2 (1968) 191-311.

[22] F. Erdogan, G. D. Gupta, T. S. Cook, Numerical solution of singular integral equations, in: G. C. Sih (Ed.), Mechanics of Fracture: Methods of analysis and solutions of crack problems, Springer, 1973, pp. 368-425.

[23] A. R. Zak, M. L. Williams, Crack point stress singularities at a bi-material interface.

[24] J. Dundurs, Edge-bonded dissimilar orthogonal elastic wedges under normal and shear loading, Journal of applied mechanics 36 (3) (1969) 650-652.

[25] F. Erdogan, G. D. Gupta, The inclusion problem with a crack crossing the boundary, International Journal of Fracture 11 (1) (1975) 13-27.

[26] A. D. Polyanin, A. V. Manzhirov, Handbook of integral equations, CRC press, 2008.

[27] G. D. Gupta, F. Erdogan, The problem of edge cracks in an infinite strip, Journal of applied Mechanics 41 (4) (1974) 1001-1006. 
[28] S. Bachu, D. B. Bennion, Experimental assessment of brine and/or $\mathrm{CO}_{2}$ leakage through well cements at reservoir conditions, International Journal of Greenhouse Gas Control 3 (4) (2009) 494-501.

[29] J. W. Hutchinson, Z. Suo, Mixed mode cracking in layered materials, Advances in applied mechanics 29 (63) (1992) 191. 